\title{
Network model for thermal conductivities of unidirectional fiber-reinforced composites
}

\author{
Yang Wang* ${ }^{*}$ Chaoyi Peng, Weihua Zhang \\ College of Aerospace Science and Engineering, National University of Defense Technology, Changsha 410073, PR China
}

\begin{abstract}
An empirical network model has been developed to predict the in-plane thermal conductivities along arbitrary directions for unidirectional fiber-reinforced composites lamina. Measurements of thermal conductivities along different orientations were carried out. Good agreement was observed between values predicted by the network model and the experimental data; compared with the established analytical models, the newly proposed network model could give values with higher precision. Therefore, this network model is helpful to get a wider and more comprehensive understanding of heat transmission characteristics of fiberreinforced composites and can be utilized as guidance to design and fabricate laminated composites with specific directional or specific locational thermal conductivities for structures that simultaneously perform mechanical and thermal functions, i.e. multifunctional structures (MFS).
\end{abstract}

Keywords: network model; thermal conductivity; fiber-reinforced composites; multifunctional structures (MFS)

(C) Wroclaw University of Technology.

\section{Introduction}

Fiber reinforced composites have found extensive applications, e.g. aerospace, automotive, shipbuilding, nuclear and other high-technology industrial fields, and still the use is on the rise. Most composites developed in earlier years were fabricated only to enhance mechanical properties, namely strength, stiffness, toughness and suchlike. However, more and more additional requirements of other characteristics, such as electrical, magnetic etc., especially thermal property (e.g. ability to withstand high temperatures or remove heat rapidly from a certain location or along a specific direction) are put forward, especially when multifunctionality is increasingly getting more and more appealing and important to many structural systems. For most proposed multifunctional structures (MFS), devices or equipment that have certain functionalities are embedded in, or bonded to, the once standalone mono-functional structural parts $[1,2]$, introducing severe thermal problems to the whole MFS. In structural batteries, for instance, gel polymer lithium-ion (GPLI) batteries are

*E-mail: wallace998@sina.cn inserted into satellite primary structures as a part of the sandwich core so as to directly bear some mechanical load in addition to electrical power storage and supplying. Since heat would be generated and released during GPLI batteries' charging/discharging it is particularly important to keep a proper temperature for the embedded batteries' normal work by heat dissipation and thermal management of MFS. Ensuring proper temperature envelope, thus, becomes an inevitable consideration and sometimes a challenging problem. However, conventional thermal control approaches used in current spacecrafts, such as radiator and pipes etc. may prove to be effective but tend to make the overall system too bulky, complex and expensive by adding parasitic weight and additional power requirements, therefore, decreasing or even eliminating the benefits firstly brought by multifunctional design. At this time, if the structural composite itself could simultaneously offer excellent thermal properties besides the existing satisfactory mechanical performance, it would be helpful to solve the introduced thermal problem and offer the possibility of designing stronger, lighter and multi-functional structures. Therefore, it is important and useful to have further understanding 
of fiber-reinforced composites' physical and especially thermal properties.

The effective thermal conductivities and other thermo-physical properties of unidirectional fiberreinforced composites have been a topic of considerable investigations by theoretical [3-9], numerical [10-15], experimental [16, 17] and mixed [18, 19] means during the past decades, involving factors, such as properties of each phase, fiber volume fraction, geometrical arrangements and interface resistance etc. While the published literature is quite extensive, most of them studied the thermal conductivities parallel and perpendicular to the reinforcing fibers using a rule of mixture, namely, parallel and serial models, respectively. And only in literatures [6] and [16] models that could be used to calculate in-plane thermal conductivities along arbitrary directions were introduced, but it is worthwhile noting that the two models give different results and besides, neither of them has yet been proved by experimental data.

Right now, what is lacking is a systematic and comprehensive understanding of thermal conductivity properties of unidirectional fiber-reinforced composites, which could be utilized either to fabricate laminated fiber-reinforced composites with specific directional thermal conductivities or to carry out 'what if' types of simulation to assess the effect of fiber content and orientation in individual lamina on the global conductivities, and which once has been known, can especially be employed in the designing of MFS that involves laminated fiber reinforced composites with both superior mechanical and thermal characteristics. Meanwhile, it would also be helpful to tailor the thermal performance of fiber-reinforced composites for specific target locations as well as for specific target directions, depending on the forthcoming thermal loads.

In order to determine the in-plane thermal conductivities along arbitrary directions in unidirectional fiber-reinforced composites, an empirical network model was proposed and developed. Comparison with experimental data and other known analytical models showed that the established network model could offer values with high precision and, thus, can be used to design MFS with satisfactory thermal conductivities as well as excellent mechanical performances.

\section{Models}

The thermal properties of fiber-reinforced composites are a function of the properties of each of the constituents. In the analysis it is always assumed that fiber and matrix are homogeneous and isotropic materials, and the composites are macroscopically homogeneous. Besides, the thermal contact resistance between the fiber and the matrix is negligible, the composites are free of voids and the fibers are distributed uniformly and arranged periodically in the matrix.

Then, for a single fiber-reinforced composite ply, the thermal conductivity in the longitudinal direction (parallel to the fiber, named as $\mathrm{k}_{1}$ ) and transverse direction (perpendicular to the fiber, named as $\mathrm{k}_{2}$ ) may be calculated using rules of mixture expressed by the following equations:

$$
\begin{gathered}
k_{1}=k_{f} v_{f}+k_{m}\left(1-v_{f}\right) \\
k_{2}=\frac{1}{\frac{v_{f}}{k_{f}}+\frac{1-v_{f}}{k_{m}}}
\end{gathered}
$$

where $v_{f}$ is the fiber volume fraction, $\mathrm{k}_{f}$ is the fiber conductivity and $\mathrm{k}_{m}$ is the matrix material conductivity.

These two principle conductivities, i.e. $\mathrm{k}_{1}$ and $\mathrm{k}_{2}$ can then be used to calculate arbitrary direction in-plane thermal conductivity (named as $\mathrm{k}_{\theta}$ ) for individual lamina as was done in [6]:

$$
k_{\theta}=\left|k_{1} \cos \theta\right|+\left|k_{2} \sin \theta\right|
$$

where $\mathrm{k}_{\theta}$ is the thermal conductivity along a direction inclined at an angle $\theta$ to fiber orientation within the composite ply. For convenience, this model will be labeled as Model_1 in the following text.

However, the calculation equation for the inplane thermal conductivity of unidirectional fiberreinforced composites was derived in [16] as:

$$
k_{\theta}=k_{1} \cos ^{2} \theta+k_{2} \sin ^{2} \theta
$$


where all the items have been defined before. Apparently, this model is much different from the above one. What is more, both of them have not reported a comparison with experimental data. And also, this model will be regarded as Model_2 in the under mentioned text.

In actual fact, the parallel model, i.e. the model depicted by equation 2, illustrates an instance in that both the two phases, namely, the fiber and the matrix, within the composite are separated completely in the direction, along which heat is transmitted. The global conductivity is determined by that of the other one if one of these two phases' conductivities goes to infinity. Hence, the global conductivity will be infinitesimal if one of the two phases' conductivities gets close to zero.

However, as for the serial model, which is illustrated in equation 1, another case is presented, namely, the two constituents of the composite are connected completely along the heat transmitting direction. When one conductivity goes to infinity, no matter if it is that of the fiber or that of the matrix, so will the global conductivity of the composite. Besides, the global conductivity can be determined by that of the other one if one of these two phases' conductivities gets close to zero.

Generally, it is found that the serial model will give a conservative and smaller value while the result of the parallel model is always exaggerated and larger. Actually, the real thermal conductivity of composites along arbitrary in-plane direction locates between these two models, namely, is a combination that integrates the contributions of the two models. Therefore, an empirical network model, represented by the following equation, can be used to depict this global thermal conductivity:

$$
k_{\theta}=f\left(k_{1}\right)+f\left(k_{2}\right)=k_{1}^{G(\theta)} \cdot k_{2}^{1-G(\theta)}
$$

where $G(\theta)$ is the network index influenced by fiber fraction, internal voids and mainly determined by the angle of inclination $\theta$ of heat flow with respect to fiber orientation. It is usual to choose a polynomial to fit data that are nonlinear and in view of the facts that the polynomial becomes more 'squiggly' as its order increases. However, if the order is too low it will hardly capture the obvious trends in the data, and, thus, considering its flexibility and ability to model simple trends, the second-order polynomial is used to fit this network index, $G(\theta)$, which is expressed as:

$$
G(\theta)=a_{0}+a_{1} \theta+a_{2} \theta^{2}
$$

where $\theta$ has been defined before, and $\mathrm{a}_{0}, \mathrm{a}_{1}, \mathrm{a}_{2}$ are undetermined coefficients, and all these four parameters can be worked out with the 'help' of experimental data using least-squares polynomial fitting algorithm.

Once upon this network model has been set up, we can not only get a deep comprehension of thermal properties of CFRP composites but also use it to fabricate laminated CFRP composites with specific directional thermal conductivities. With additional assumption that all individual lamina have identical thickness, then the in-plane thermal conductivity of laminated CFRP composite can be computed using:

$$
K_{\theta}=\frac{1}{N} \sum_{i=1}^{N} k_{i, 1}^{G\left(\theta_{i}\right)} \cdot k_{i, 2}^{1-G\left(\theta_{i}\right)}
$$

where $\mathrm{K}_{\theta}$ is the in-plane thermal conductivity of laminated CFRP composite along specific direction, $\mathrm{N}$ is the total number of lamina in the laminated composite, the subscript $i$ denotes each individual lamina, $\theta_{\mathrm{i}}$ signifies the angle between the $\mathrm{i}^{\text {th }}$ ply and the $\mathrm{K}_{\theta}$ direction $\left(\mathrm{k}_{\mathrm{i}, 1}\right.$ and $\mathrm{k}_{\mathrm{i}, 2}$ are longitudinal and transverse thermal conductivities of the $i^{\text {th }}$ ply).

\section{Experimental}

\subsection{Materials}

Different fiber/matrix combinations were adopted, namely, M40J (high modulus pitch carbon fiber, provided by Toray Corp.), T700 (high strength PAN based carbon fiber, produced by Toray Corp.), E648 (epoxy resin, manufactured in Shanghai Resin Corp.) and E23A (epoxy resin, offered by Sky-bird New-Tec Corp.). Table 1 shows the properties of the fibers used. They were chosen partly because of the aim to fabricate specimens of different material systems to learn more about 
fiber-reinforced composites and partly because these two kinds of carbon fiber are commonly used in aerospace industry and this research is a part of the program on multifunctional structural battery (MFSB) for satellite application, and of course a simple and natural way to solve the thermal problem introduced by MFSB will be the first choice.

\subsection{Thermal conductivity measurements}

Thermal conductivities of a unidirectional fiberreinforced composite were measured using the laser flash method, in which a laser beam was flashed on the top surface of a cylindrical specimen; meanwhile the temperature change at the bottom surface of the specimen was monitored and recorded. Based on the measured temperature history, the half time $\mathrm{t}_{\frac{1}{2}}$ (time for the temperature rise up to the half of the maximum temperature) was determined, which in turn was used to compute specimen thermal diffusivity, $\alpha$, using the following equation:

$$
\alpha=0.1388 \frac{L^{2}}{t_{1 / 2}}
$$

where $\mathrm{L}$ is the specimen length. Based on the thermal diffusivity, thermal conductivity can be calculated using the following equation:

$$
k=\alpha c \rho
$$

where $\mathrm{c}$ is the specific heat capacity and $\rho$ is the density. Generally, the heat capacity can be measured by DSC method or sometimes got directly from related literatures etc. And in this study, the coefficient comparison method was adopted.

In this paper, disc-shaped specimens, approximately $20 \mathrm{~mm}$ in diameter, were produced from the molded plates with the aid of a disc cutter. Then the diameters were reduced to $12.6 \mathrm{~mm}$ and the thickness of each disc was reduced to the required values by grinding and polishing, about 2 to $4 \mathrm{~mm}$. A list of the specimens prepared and investigated is summarized in Table 2. The specimens were prepared with high quality and the test conditions were controlled strictly to make the test accuracy as high as possible. Thermal conductivities were measured using LFA-447 Quick Thermal Conductivity Meter (NETZSCH, Germany). As for the test parameters, the sample-point number was set to be 2000, the pulse power, which was adjustable, was set to $10 \mathrm{~J}$, and the duration was selected to ensure that the curve length behind the pulse was 10 to 15 times to that of the $t_{\frac{1}{2}}$. Besides, the magnitude of the amplifier was chosen to make sure that the signal difference would locate between $1 \mathrm{~V}$ and $10 \mathrm{~V}$.

Note: the number of plies in each laminated specimen was chosen thoroughly so as all specimens had identical thickness that fitted the examination requirement and each specimen were tested three times at $298 \mathrm{~K}, 323 \mathrm{~K}, 373 \mathrm{~K}$ and $423 \mathrm{~K}$. The fiber volume was calculated according to the micrographs of the specimens obtained using scanning electron microscope (FEI Quanta200 , Netherlands), and the void content was measured by the Archimedes water-immersion method.

\section{Results and discussion}

To determine the experimental parameters so as to get the empirical network model, necessary mathematical process have been performed.

After logarithmic transformation of equation 5 to get rid of the exponential term:

$\ln k_{\theta}=\ln \left(k_{1}^{G(\theta)} \cdot k_{2}^{1-G(\theta)}\right)=\ln k_{2}+\left(\ln k_{1}-\ln k_{2}\right) G(\theta)$

We get:

$$
G(\theta)=\ln \frac{k_{\theta}}{k_{2}} / \ln \frac{k_{1}}{k_{2}}
$$

After substituting experimental data into this equation a list of network indices, shown in Table 3, has been obtained.

Note: as the relations between thermal conductivity of CFRPs and working temperature have not been investigated or discussed in this paper, all the listed experimental data refer to $298 \mathrm{~K}$.

Using the polynomial fitting, as depicted in equation 6 , for the three kinds of composites, we get the values of undetermined coefficients for network indices shown in Table 4.

Fig. 1 shows the experimentally obtained network indices and their polynomial fitting with 
Table 1. Properties of carbon fibers from product data sheets.

\begin{tabular}{ccccc}
\hline \multirow{2}{*}{ Carbon fiber Type Density $\left(\mathrm{g} / \mathrm{cm}^{3}\right)$} & \multicolumn{3}{c}{ Thermal conductivity $(\mathrm{W} / \mathrm{m} \mathrm{K})$} \\
\cline { 4 - 5 } & & & longitudinal & transversal \\
\hline \hline M40J & $6 \mathrm{~K}$ & 1.75 & 40 & - \\
T700 & $12 \mathrm{~K}$ & 1.80 & 8 & - \\
\hline
\end{tabular}

Table 2. The specimens.

\begin{tabular}{|c|c|c|c|c|c|}
\hline $\begin{array}{l}\text { Specimen } \\
\text { designation }\end{array}$ & Constituent & $\begin{array}{l}\text { Fiber orientation } \\
\text { angle (deg) }\end{array}$ & $\begin{array}{l}\text { Direction of thermal conductivity } \\
\text { measurement (deg) }\end{array}$ & $\begin{array}{c}\text { Fiber volume } \\
(\%)\end{array}$ & $\begin{array}{l}\text { Void content } \\
\quad(\%)\end{array}$ \\
\hline A1 & M40J/E648 & lamina & $0,30,45,60,90$ & 56.3 & 0.1 \\
\hline A2 & M40J/E648 & $0 / 90$ & $0,45,60$ & 54.5 & 0.2 \\
\hline A3 & M40J/E648 & $0 / 45 /-45 / 0$ & $0,45,60$ & 54.5 & 0.2 \\
\hline B1 & T700/E648 & lamina & $0,30,45,60,90$ & 64.2 & 0.1 \\
\hline B2 & T700/E648 & 0/90 & $0,45,60$ & 64.2 & 0.2 \\
\hline B3 & T700/E648 & $0 / 45 /-45 / 0$ & $0,45,60$ & 62.7 & 0.1 \\
\hline $\mathrm{C} 1$ & T700/E23A & lamina & $0,30,45,60,90$ & 63.3 & 0.1 \\
\hline $\mathrm{C} 2$ & $\mathrm{~T} 700 / \mathrm{E} 23 \mathrm{~A}$ & $0 / 90$ & $0,45,60$ & 60.2 & 0.1 \\
\hline $\mathrm{C} 3$ & T700/E23A & $0 / 45 /-45 / 0$ & $0,45,60$ & 63.7 & 0.2 \\
\hline
\end{tabular}

respect to the variation of the angle of inclination to the fiber direction. It can be clearly seen that the selected two-order polynomial fitting (which belongs to nonlinear fitting) simulates the experimental results well. Thus, the network indices together with equation 6 could be applied for these three kinds of composites at the current situation (including manufacture process, fiber volume, void content and working temperature etc.), namely:

$$
G(\theta)=0.9907-0.0432 \theta-0.3712 \theta^{2}, \theta \in
$$
$\left(0, \frac{\pi}{2}\right)$ for $\mathrm{M} 40 \mathrm{~J} / \mathrm{E} 648$ system;

$$
G(\theta)=0.9959-0.0571 \theta-0.3662 \theta^{2}, \theta \in
$$
$\left(0, \frac{\pi}{2}\right)$ for T700/E648 system;

$$
G(\theta)=0.9878-0.0799 \theta-0.3454 \theta^{2}, \theta \in
$$
$\left(0, \frac{\pi}{2}\right)$ for $\mathrm{T} 700 / \mathrm{E} 23 \mathrm{~A}$ system.

With the determination of these model parameters, namely, network indices and coefficients, the proposed network model could be set up.

Fig. 2 presents the in-plane thermal conductivities along arbitrary directions for unidirectional fiber-reinforced composites computed by network model and models proposed in literatures [6] and [16] compared with the experimental data. It can be qualitatively observed that with the increase of the angle between thermal flow and fiber orientation, the in-plane thermal conductivity decreases nonlinearly and this trend is successfully reflected by all the three models. However, the proposed network model gives the best simulation. Besides, specimens with high modulus fibers have stronger heat transmission ability than those made up of high strength fibers.

Although this network model could offer more precise results, its formulation needs some experimental data as a fitting basis apart from the parallel and serial models. To validate the effectiveness of the proposed network model, a direct way would be to compare the experimental values of thermal conductivity along some other directions with the values predicted by the network and other analytical models. However, as CFRP composite are generally used in laminated form for engineering applications, in this paper, the evaluation of this network model was carried out by testing typical engineering 
Table 3. The obtained network indices.

\begin{tabular}{|c|c|c|c|c|c|c|}
\hline Direction $(\mathrm{deg})$ & & 0 & 30 & 45 & 60 & 90 \\
\hline \multirow{3}{*}{ Thermal conductivity $\mathrm{W} / \mathrm{m} \cdot \mathrm{K}$} & A1 & 23.20 & 15.25 & 11.86 & 7.57 & 1.81 \\
\hline & B1 & 10.22 & 7.97 & 6.61 & 4.77 & 1.96 \\
\hline & $\mathrm{C} 1$ & 9.93 & 7.14 & 6.24 & 4.57 & 1.78 \\
\hline \multirow{3}{*}{ Network index } & A1 & 1 & 0.83551 & 0.73696 & 0.56094 & 0 \\
\hline & B1 & 1 & 0.84942 & 0.73613 & 0.53857 & 0 \\
\hline & $\mathrm{C} 1$ & 1 & 0.80811 & 0.72973 & 0.54853 & 0 \\
\hline
\end{tabular}
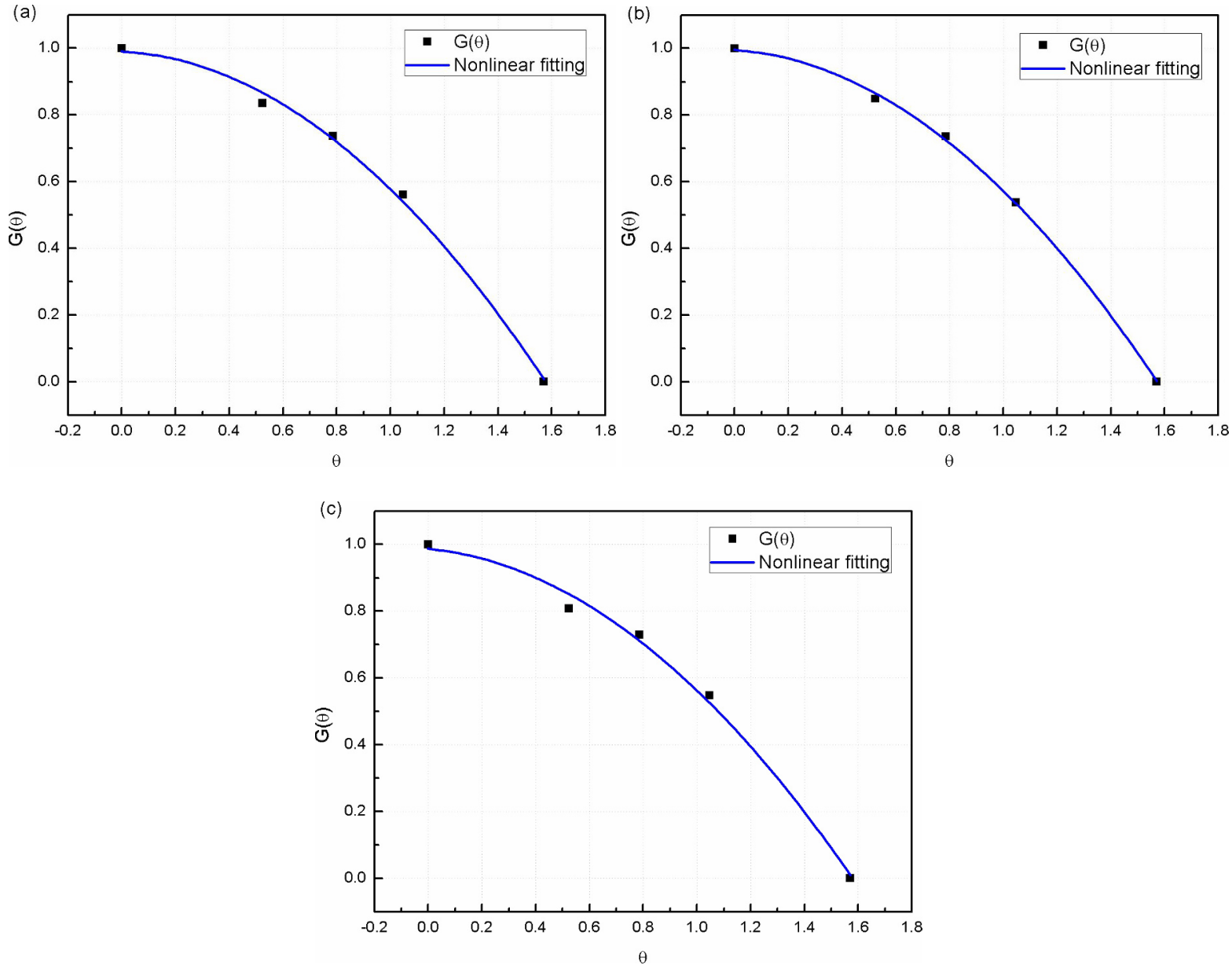

Fig. 1. Experimental network indices and their nonlinear fitting for the three kinds of composites: (a) nonlinear fitting for A1; (b) nonlinear fitting for B1; (c) nonlinear fitting for $\mathrm{C} 1$.

Table 4. Coefficients for network indices.

\begin{tabular}{cccc}
\hline & $a_{0}$ & $a_{1}$ & $a_{2}$ \\
\hline \hline M40J/E648 & 0.9907 & -0.0423 & -0.3712 \\
T700/E648 & 0.9959 & -0.0571 & -0.3662 \\
T700/E23A & 0.9878 & -0.0799 & -0.3454 \\
\hline
\end{tabular}

CFRP laminates, namely, [0/90] and [0/45/-45/0] that are listed in Table 2 as specimens A-2, A-3, B-2, B-3, C-2 and C-3.

Fig. 3 illustrates the values predicted by the proposed network model in comparison with experimental data and FEM results for these 

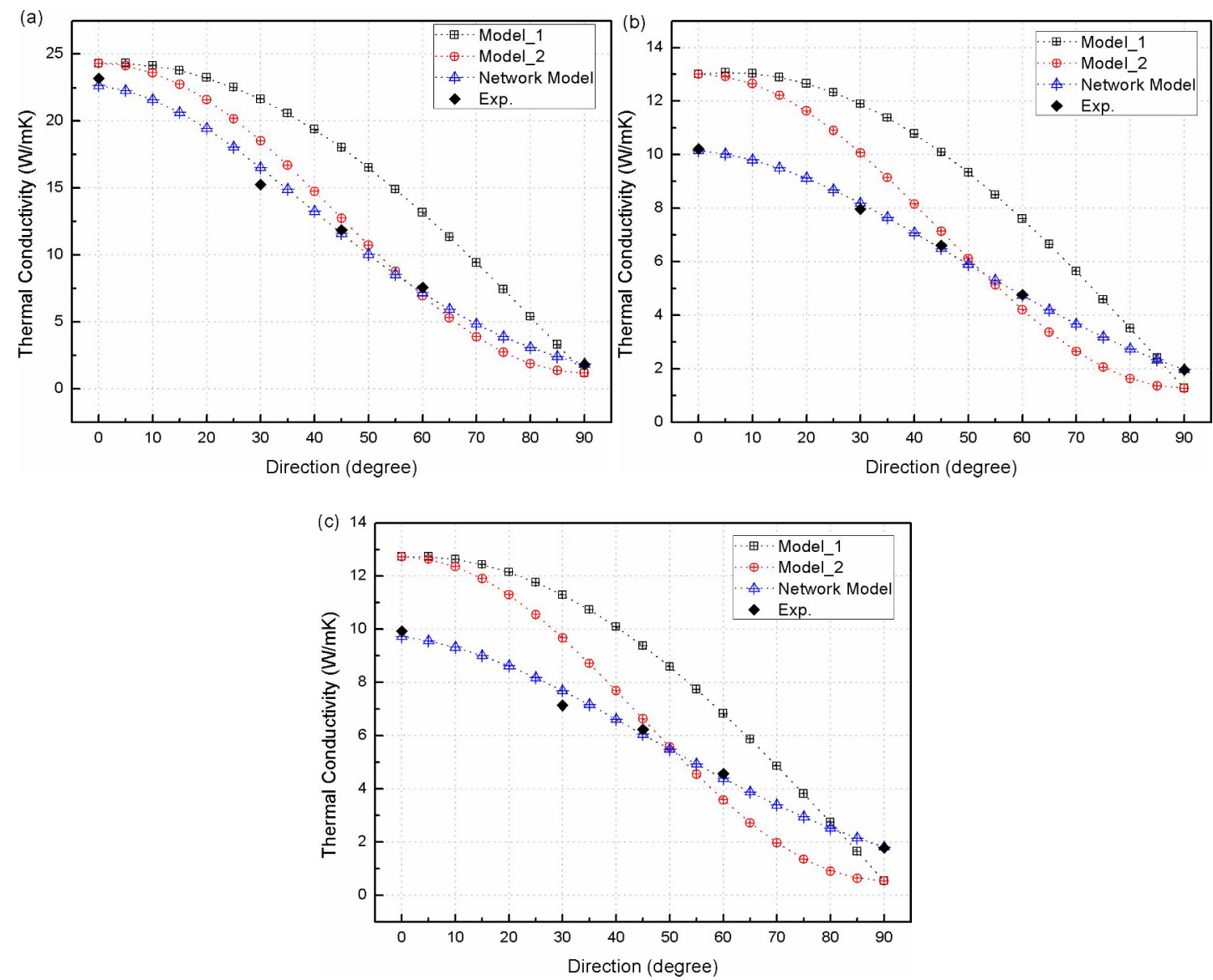

Fig. 2. Comparison between Network model and the established models: (a) A1 thermal conductivities in different directions; (b) B1 thermal conductivities in different directions; (c) $\mathrm{C} 1$ thermal conductivities in different directions.
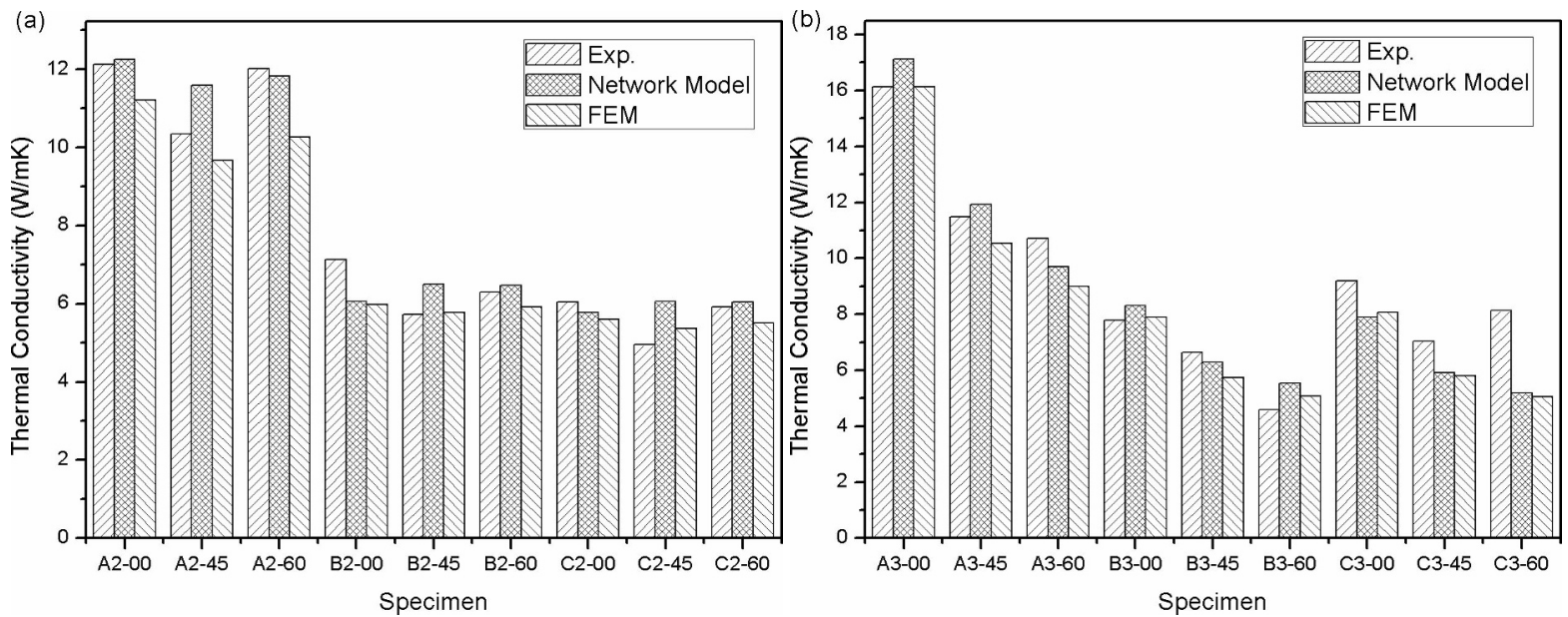

Fig. 3. Comparison between Network model and the established models: (a) for [0/90] laminate; (b) for [0/45/45/0] laminate. 
A/B/C-2/3 specimens, along different directions. The FEM analysis selected in this comparison is the same as that used in [12]. It can be seen that, for most instances, the network model could give excellent predictions that are close to the experimental results, indicating its effectiveness in predicting thermal conductivities along arbitrary directions within laminated fiber-reinforced composites, thus, the model can be used as an aid in fabricating laminated fiber-reinforced composites, with specific directional thermal conductivities.

\section{Conclusions}

An empirical network model has been proposed to predict the in-plane thermal conductivities along arbitrary directions for unidirectional fiberreinforced composites. Comparison with existing models and experimental data has also been drawn. Good agreement was observed between predicted and measured values for the current fiber and resin systems, indicating the effectiveness of this network model for typical CFRP composites, which would be helpful to gain better understanding of their heat transmitting properties. The model can be used to tailor the thermal performance of fiberreinforced composites for specific target locations as well as for specific target directions and, first of all, in the design of multifunctional composite structures.

\section{Acknowledgements}

The authors would like to acknowledge the support of the Cooperative Zhuzhou Times New Material Technology Corp. Ltd. for this work.

\section{References}

[1] Aglietti G.S., Schwingshackl C.W., Roberts S.C., Shock Vib., 39 (2007), 381.
[2] Gibson R.F., Compos. Struct., 92 (2010), 2793.

[3] Spring G.S., Tsai S.W., J. Compos. Mater., 1 (1967), 166.

[4] Hasselman D.P.H., Johnson L.F., J. Compos. Mater., 21 (1987), 508.

[5] Pitchumani R., Yao S.C., J. Heat Trans.-T. ASME., 113 (1991), 788.

[6] KulKarni M.R., BRady R.P., Compos. Sci. Technol., 57 (1997), 277.

[7] Yan P., Jiang C.P., Song F., Xu X.H., Chinese J. Aeronaut., 23 (2010), 54.

[8] Zou M.Q., Yu B.M., Zhang D.M., J. Phys. D Appl. Phys., 35 (2002), 1867.

[9] Wang J.F., Carson J.K., North M.F., Cleland D.J., Int. J. Heat Mass Tran., 49 (2006), 3075.

[10] Noor A.K., Shah R.S., Compos. Struct., 26 (1993), 7.

[11] Veyret D., Cioulachtuian S., Tadrist L., PanTALONI J., J. Heat Trans.-T. ASME, 115 (1993), 866.

[12] Islam M.R., Pramila A., J. Compos. Mater., 33 (1999), 1699.

[13] Rocha P.A., Cruz M.E., Numer. Heat Tr. A-Appl., 39 (2001), 179.

[14] Graham S., McDowell D.L., J. Heat Trans.-T. ASME, 125 (2003), 383.

[15] SiHn S., RoY A.K., J. Compos. Mater., 45 (2011), 1245.

[16] Pilling M.W., Yates B., Black M.A., J. Mater. Sci., 14 (1979), 1326

[17] Thornburg J.D., Pears C.D., ASME paper 65WA/HT-4 (1965).

[18] Rolfes R., Hammerschmidt U., Compos. Sci. Technol., 54 (1995), 45.

[19] Turias I.J., Gutiérrez J.M., Galindo P.L., Compos. Sci. Technol., 65 (2005), 609. 